\title{
AN ANTIPROTON-DRIVEN MAGNETICALLY INSULATED INERTIAL FUSION PROPULSION SYSTEM
}

\author{
Terry Kammash and David L. Galbraith \\ Department of Nuclear Engineering \\ The University of Michigan \\ Ann Arbor, MI 48109 \\ (313 764-0205
}

\author{
Brice N. Cassenti \\ United Technologies Research Center \\ 411 Silver Lane \\ Ease Hartford, CT 06108 \\ (203) $727-7460$
}

\begin{abstract}
$\underline{\text { Abstract }}$
The magnetically Insulated Inertial Confinement Fusion (MICF) reactor, in its initial conception, concepts of a target in the form of a metal shell whose inner surface is coated with a fusion fuel which is ignited by an incident laser beam that enters the pellet through a hole. A very strong magnetic field, generated when the surface is ablated by the incident laser beam, provides thermal insulation of the wall from the hot plasma, and allows the plasma to burn longer thereby generating a larger energy amplification. When ejected through a magnetic nozzle the plasma can provide a very large specific impulse if MICF is utilized as a propulsion device. For application to space travel, however, the mass of the laser and associated power supply may prove to be prohibitively large and another driver should be considered in its place. In this paper we examine the potential use of antimatter annihilation reactions along with a fissionable component to generate the energy needed to initiate the fusion reactions. We find that a modest amount of antiprotons impinging on a tiny fissioning "spark" can ignite the pellet and produce specific impulses in excess a hundred thousand seconds.
\end{abstract}

\section{INTRODUCTION}

One of the most novel approaches to fusion power is the magnetically Insulated Inertial Confinement Fusion (MICF) concept (Hasegawa 1986) illustrated in Figure 1. It combines the favorable aspects of both magnetic and inertial fusion in that physical containment of the burning plasma is provided by a metallic shell, while insulation of its thermal energy from material walls is provided by a strong, self-generated magnetic field. In contrast to conventional, implosion-type inertial confinement systems, MICF utilizes a plasma that is formed inside the target pellet as result of ablation of the fuel-coated inner wall by a laser (or a particle) beam that enters the target through a hole. Since the confinement time in this system is dictated by the sound speed in the shell (Kammash and Galbraith 1989) (as opposed to the sound speed in the plasma) a significant enhancement $\left(\sim 10^{2}\right)$ of the lifetime of plasma energy in MICF is achieved over implosion-type systems due to the reduction of the shell temperature arising from the thermal insulation provided by the magnetic field. Moreover, the coupling of laser energy to the plasma is more efficient in MICF since the energy is put directly into the plasma rather than in the imploding pusher as it is in implosion-type inertial fusion. These properties allow MICF to generate very large energy multiplication, which manifests itself in a high temperature plasma which in turn could yield very large exit velocities if allowed to be ejected through a magnetic nozzle. These velocities translate into large specific impulses when MICF is utilized as a propulsion device; a property that renders it especially attractive for space exploration. A major obstacle in this regard is the prohibitively massive laser system and associated power supply, and the success of MICF as an effective space propulsion system rests heavily on the availability of a driver that is light and compact. Because of their very large energy density, antiproton annihilation reactions could provide the answer if and when the technology of making, staring, and manipulating antihydrogen becomes realizable. In this paper we explore the use of antihydrogen to drive MICF, and with the aid of some preliminary analysis we estimate the number of such particles needed to ignite.

\section{ANTI-PROTON DRIVER}

We introduce an anti-proton beam through a hole on the side of the target as illustrated in Figure 2, and make it strike a hemisphere of fissionable material such as $\mathrm{U}^{235}, \mathrm{U}^{238}$ or $\mathrm{Pu}^{239}$. The fusion fuel is surrounded by a shell which we choose to be $\mathrm{U}^{238}$, and that in turn is surrounded by the tungsten shell which provides the physical containment of the system. The energy of the antiproton beam is chosen so that the annihilation reaction takes place at the surface of the hemisphere. Annihilation of the anti-protons ionizes the fuel above the hemisphere, and 


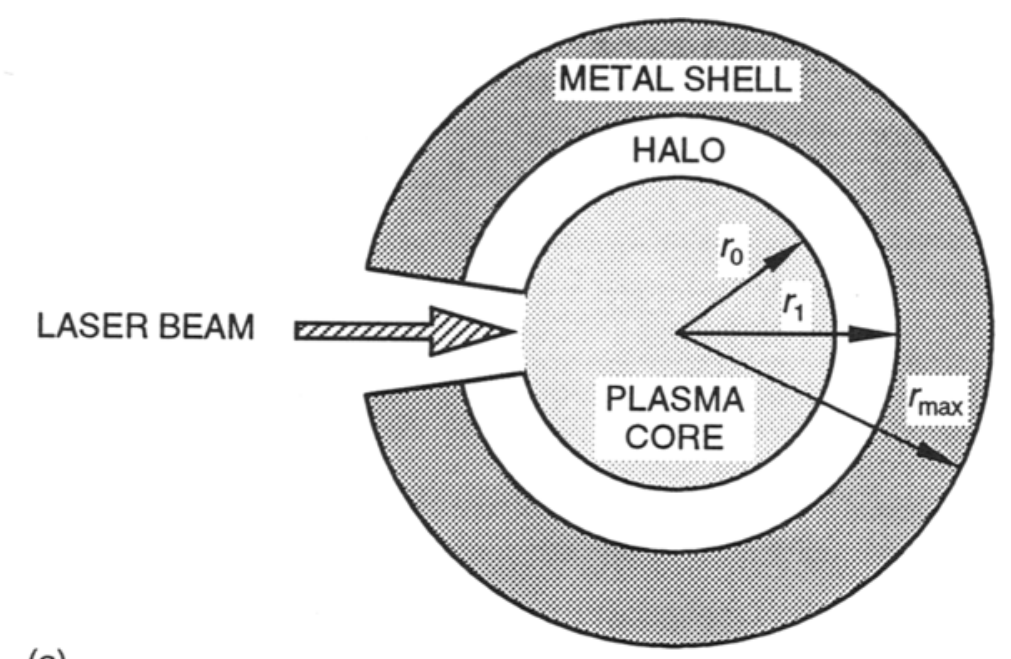

(a)

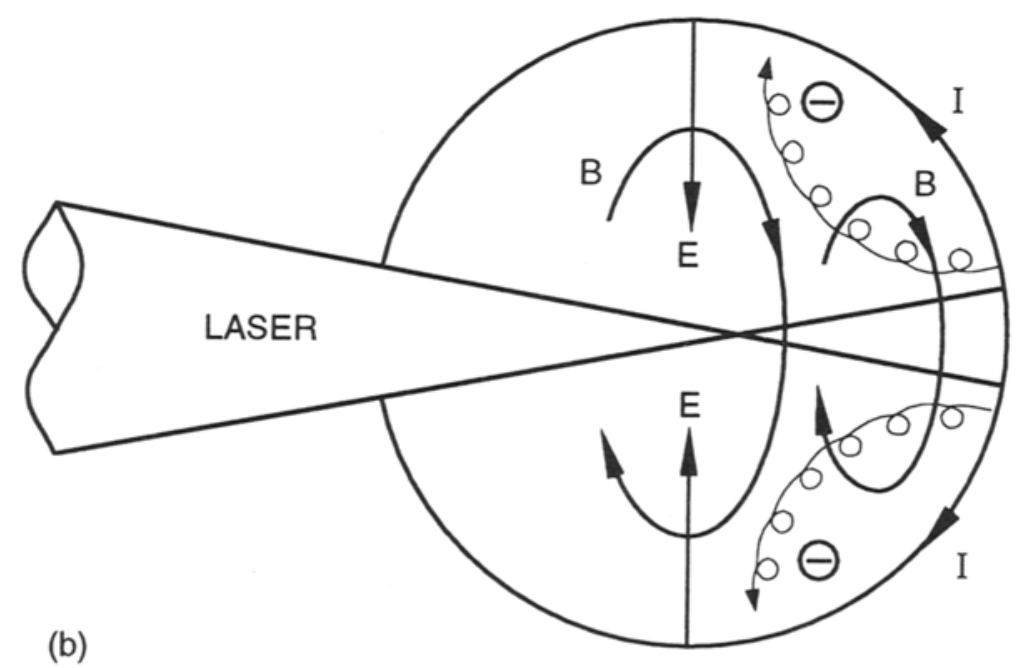

FIGURE 1. Schematic Diagram of (a) Plasma Formation and (b) Magnetic Field Formation in MICF.

the resulting fuel ions fill the empty core of the pellet with the fusion plasma. As noted earlier, a strong magnetic field is generated whose strength (Aslan and Kammash 1994), on the average, is maximum at the boundary between the hot core and the cold plasma (halo region) that separates it from the outer shell. This field traps the charged particles generated in the system, namely the fusion fuel ions and electrons, as well as the muons and pions and the fission fragments generated by the fissioning of the hemisphere. These charged particles heat the core to fusion temperatures, and the uranium shell on the inside layer of the outer hell generates and/or reflects some neutrons back into the fuel. The fusion burn in the core will last for the length of time it takes the shock wave, initiated at the plasma-wall interface to traverse the thickness of the tungsten shell.

In the absence of the uranium shell, an analytical model that describes the dynamics of the system was formulated (Cassenti et al. 1994) and solved to produce the performance of a rocket that employs an anti-hydrogen driven MICF. It was shown that a plasma of $1.25 \times 10^{21} \mathrm{~cm}^{-3}$ density at a temperature of $83 \mathrm{keV}$ is produced in the core when an anti-matter pulse containing $3 \times 10^{9}$ anti-protons was applied for $30 \mathrm{~ns}$. An ideal specific impulse for the system was determined on the assumption that all the fusion alpha particle (produced by the deuterium-tritium reaction) energy is emitted with the total mass of the pellet which was taken to be $3.5 \mathrm{~g}$. The results are shown in Table 1 for different burn-up fractions. When a uranium shell is included in the calculation to see if sufficient compression of plasma can be achieved as the magnetic field decays it was found that a $0.2 \mathrm{~cm}$ thick $\mathrm{U}^{238}$ shell may produce enough pressure for 


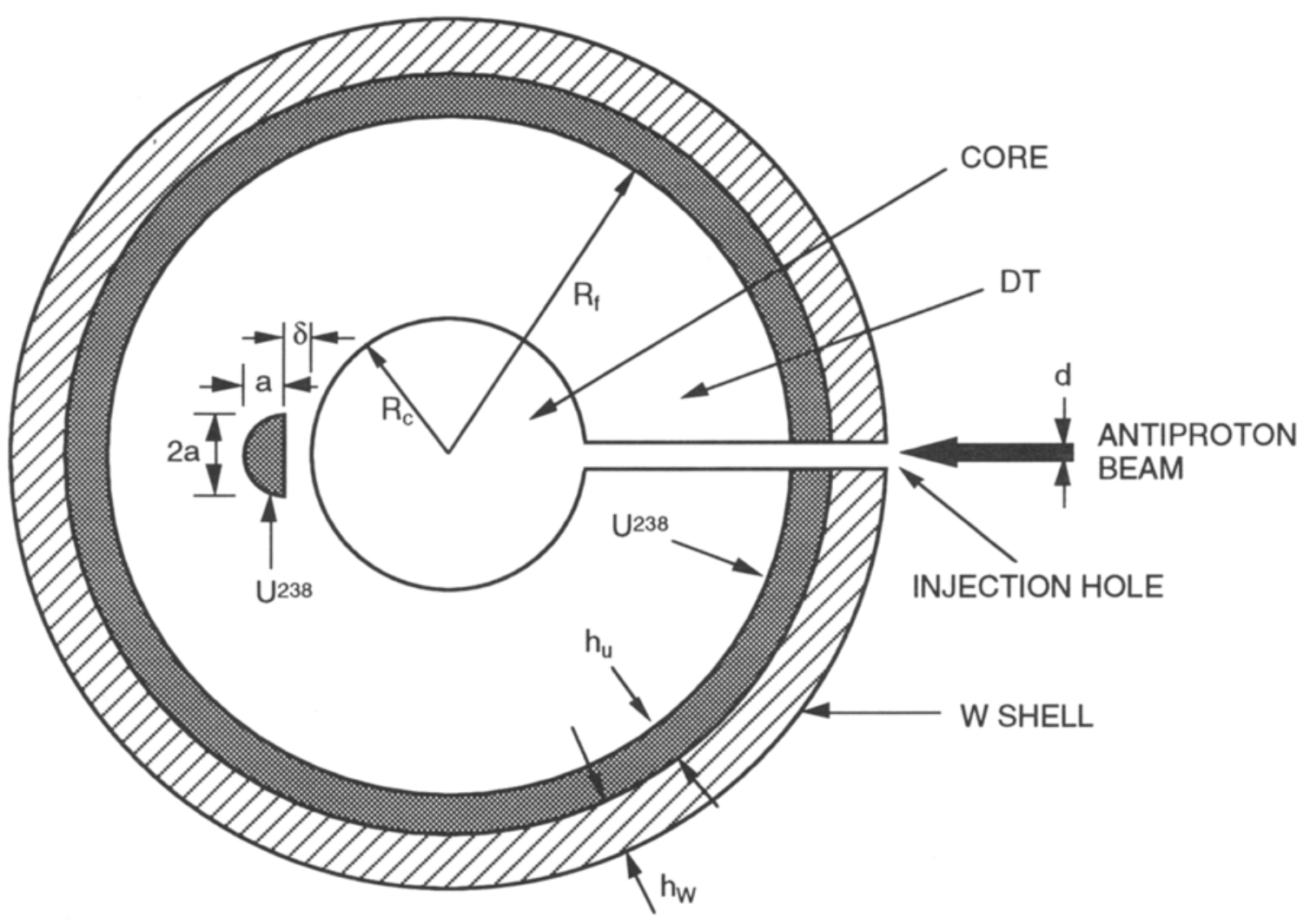

Pellet Construction and Geometry

FIGURE 2. Antiproton driven MICF.

the duration of the confinement time to sustain an energy production commensurate with the above-noted propulsion charcteristics. These results are also given in Table 1.

TABLE 1. Performance of an Anti-proton Driven MICF Pellet.

Pellet radius $R_{f}=1 \mathrm{~cm}$

Uranium shell thickness $h_{u}=0.2 \mathrm{~cm}$ $\delta=0.0075$
Fusion confinement time $=20 \mathrm{~ns}$

Uranium hemisphere radius $a=0.03 \mathrm{~cm}$

$R_{c}=0.01 \mathrm{~cm}$

\begin{tabular}{|c|c|c|}
\hline Burnup Franction & Specific Impulse $(\mathrm{s})$ & Pressure $\left(\mathrm{keV} / \mathrm{cm}^{3}\right)$ \\
\hline 0.05 & 150,000 & $2 \times 10^{23}$ \\
0.10 & 200,000 & $4 \times 10^{23}$ \\
1.00 & 600,000 & $4 \times 10^{24}$ \\
\hline
\end{tabular}

\section{CONCLUSION}

We have demonstrated in this paper the feasibility of driving an inertial confinement fusion concept with antiprotons utilizing a "spark" of fissionable material. When used as a propulsion device, we have shown that this antimatter catalyzed MICF system is capable of producing very attractive propulsion parameters such as a specific impulse of well over a hundred thousand seconds. Since the main feature of this concept is a self-generated magnetic 
field that thermally insulates the pellet wall from the hot plasma, it was necessary to establish if plasma compression can be achieved in the event that the magnetic field decays on a time scale shorter than the required confinement time. It is shown that a uranium shell of $0.2 \mathrm{~cm}$ thickness is marginally capable of providing the needed compression (Kammash and Galbraith 1989) although this aspect of the analysis needs further investigation.

\section{Acknowledgments}

This work was carried out at the Nuclear Engineering Department of The University of Michigan and at the Research Center of United Technologies.

\section{References}

Aslan, N. and T. Kammash (1994) "Plasma Dynamics in a Magnetically Insulated Target for Inertial Fusion," Fusion Technology 26:184.

Cassenti, B., T. Kammash and D. L. Galbraith (1994) "An Antiproton Catalyzed Fusion Propulsion System," AIAA 94-3354, 30th Joint Propulsion Conference, Indianapolis, IN, 27-29 June 1994.

Hasegawa, A. (1986) "Magnetically Insulated Inertial Confinement Fusion: A New Approach to Controlled Thermonuclear Fusion," Physical Review Letters 56:139.

Kammash, T. and D. L. Galbraith (1989) "A High Gain Fusion Reactor Based on the Magnetically Insulated Inertial Confinement Fusion Concept," Nuclear Fusion 29: 1079. 